\title{
Non-Linear Energy Operator for the Analysis of Intracardial Electrograms
}

\author{
C. Schilling ${ }^{1}$, M.P. Nguyen ${ }^{1}$, A. Luik ${ }^{2}$, C. Schmitt ${ }^{2}$ and O. Dössel ${ }^{1}$ \\ ${ }^{1}$ Institute of Biomedical Engineering, Universität Karlsruhe (TH), Karlsruhe, Germany \\ ${ }^{2}$ II. Medizinische Klinik, Städtisches Klinikum Karlsruhe, Karlsruhe, Germany
}

\begin{abstract}
The curative therapy of atrial fibrillation (AF) is still challenging. Although the electrophysiologists know many strategies to cure AF, the underlying mechanisms are still mostly unknown. Also the optimal ablation strategy for paroxysmal and long-lasting persistent AF is not known. Complex fractionated atrial electrograms (CFAEs) are becoming more and more important in the ablation strategies, especially for longlasting persistant AF. Automated detection and signal analysis of CFAEs is essential in supporting the physicians during the ablation procedure. The robust algorithm to locate CFAEs presented in the contribution by Nguyen, Schilling and Dössel delivers a good bases for postprocessing and signal analysis of CFAEs. It is employing a non-linear energy operator combined with thresholding. In this paper this new algorithm is tested on clinical data and compared to clinically accepted algorithms.
\end{abstract}

Keywords - Atrial Fibrillation, CFAEs, Catheter Ablation, Signal Processing

\section{INTRODUCTION}

Atrial fibrillation (AF) is the most common cardiac arrhythmia. There are two main forms of AF: paroxysmal AF and long-lasting persistent AF. Paroxysmal AF is defined as nonsustaining episodes of fibrillation shorter than seven days. It is mainly caused by focal activity in the pulmonary veins. Paroxysmal AF can be cured by electrically isolating the pulmonary veins. There are many strategies in isolating the pulmonary veins [1]. But the optimal ablation strategy is still unknown.

Under the influence of the chaotic excitation during paroxysmal AF the atrial tissue undergoes a conversion process. This process leads to a modification of the electro-physiological properties of the atrium. As a result of this process called remodelling there are more and more areas with fractionated potentials all over the atrium. The periods of AF are getting longer until AF is long-lasting persistent. A very promising approach is to map and ablate these fractionated areas. AF then can often be changed into other forms of AF, to atrial flutter or AF can even be terminated [2].

Surgical and catheter-based therapies may be used to treat and prevent recurrence of AF in certain individuals. Despite the considerable clinical experience and accumulated evi- dence from experimental data, the exact mechanism of $\mathrm{AF}$ and their elimination by catheter ablation techniques is still unknown, especially in long-lasting persistent atrial fibrillation [3] [4].

The interpretation of signals measured during an electrophysiological examination is the most important task in understanding the excitation propagation during AF. Commercial software is assisting the physicians. Analysis in time and also frequency domain is done. But not every algorithm is reasonable.

The algorithm presented in [5] is a new approach to detect complex fractionated electrograms (CFAEs) by evaluating the signals energy. The algorithm is based on the NonLinear Energy Operator defined in [6] [7] and is adapted to atrial electrograms. The main advantage of this algorithm is, that it is not delimited to a specific signal length. So also CFAEs with an activity over e.g. 12 seconds can be detected and then preprocessed. Based on this segmentation two new indices (ActivityRatio and MeanLengthofActiveSection (MLAS)) are defined to evaluate CFAEs. The physiological information of these new indices is validated by comparing it to clinically accepted algotrihms.

\section{MAterials \& Methods}

\section{A. Data Acquisition}

In coorperation with Städtisches Klinikum Karlsruhe (Academic teaching hospital of the University of Freiburg, Germany) intracardiac bipolar electrograms were examined. The data was measured during electrophysiological examinations. All patients were ablated because of persistent and long-lasting persistent atrial fibrillation (AF). All ablations were performed using the impedance based 3D navigation system EnSite $\mathrm{NavX}^{\mathrm{TM}}$ (St. Jude Medical). Complex fractionated electrograms (CFAE) were recorded before and after pulmonary vein isolation (PVI) at defined positions in the left atrium (roof, septum, anterior and posterior wall). The data was acquired with a 10 polar Lasso ${ }^{\circledR}$ catheter (Biosense Webster) over 6 seconds with a sampling rate of $1200 \mathrm{~Hz}$. In addition recordings from a multipolar catheter (8 polar, BARD inc., New Jersey, USA) in the coronary sinus delivered four leads of bipolar intracardiac ECG signals (sampling 
rate $1000 \mathrm{~Hz}$ ).

Baseline wander and low-frequency noise were eliminated from the electrograms by use of DWT-based (Discrete Wavelet Transformation) approach. The high frequency disturbance is removed by a conventional Butterworth low pass filter [8].

\section{B. Dominant Frequency}

The most common analysis of atrial fibrillation in the frequency domain is the dominant frequency (DF) analysis. It is an estimation of the atrial activation rates. If the signal is periodic and more or less sinusoidal in morphology, the dominant frequency will be related to the signal's rate. The dominant frequency will be able to reflect the periodicity of the signal better, the more the signal looks like a sine wave. The implementation of calculating the dominant frequency is done as described in [9].

1. Bandpass filtering at $40-250 \mathrm{~Hz}$

2. Absolute value (rectification)

3. Lowpass filtering at $20 \mathrm{~Hz}$

4. Windowing

5. (Zero-padding)

Finally the FFT is computed and the frequency with the highest amplitude is determined as dominant frequency.

\section{Fractionation Index (EnSite)}

CFAE-Maps were created during electrophysiolocical examinations by use of the EnSite NavX $\mathrm{X}^{\mathrm{TM}}$ software (St. Jude Medical). Stable rhythms at different regions of the left atrium are recorded with conventional catheters using the EnGuide-System. The automated algorithm of the NavX system is used to identify fractionated electrograms. A list of settings for the NavX algorithm are made as usual by the physicians (P-P sensitivity $=0.04 \mathrm{mV}$, electrogram detection: $-d V / d t$, Refractory $=40 \mathrm{~ms}$, Interpolation $=14 \mathrm{~mm}$, segment length $=5 \mathrm{~s}$, etc.). For each recorded region the automated algorithm delivers a fractionation index (CFE-Index) based on the cyclelength of distinguishable local activities in one electrogram.

\section{Non-Linear Energy Operator}

The Non-Linear Energy Operator (NLEO) is a simple algorithm to calculate the energy of a given signal. The energy of a mass-spring system with its underlying harmonic motion is proportional to the square of the frequency and to the square of the amplitude [6].

$$
E \propto A^{2} \omega^{2}
$$

Now, let $x(n)=A \cos (\Omega n+\phi)$ be a digital signal, where $\Omega=2 \pi f / f_{s}$ is the digital frequency in radians/sample, $f$ is the analog frequency and $f_{s}$ the sampling frequency. $\phi$ is the initial phase in radians and $A$ the amplitude. The values for the three unknowns $(A, \Omega$ and $\phi)$ can be obtained from three samples $\left(x_{n}, x_{n-1}\right.$ and $\left.x_{n+1}\right)$ of the signal $x(n)$. Using trigonometric identities, doing some reformulating and substitution we obtain

$$
E_{n}=x_{n}^{2}-x_{n+1} x_{n-1}=A^{2} \sin ^{2}(\Omega) \approx A^{2} \Omega^{2}
$$

This formula is unique and exact for $\Omega<\pi / 2$. To use the approximation on the right-hand side of (2) we limit $\Omega$ to $\pi / 4$, i.e. $f / f_{s}<1 / 8$. With a sampling frequency of $f_{s}=1200 \mathrm{~Hz}$ the highest frequency in the signal must be smaller than $150 \mathrm{~Hz}$.

The NLEO is used to segment the given signal by interpreting its frequency and amplitude (1). According to [5] the following processing is done

1. Denoising and Baseline Wander Removal

2. NLEO

3. Gaussian Lowpass Filtering

4. Adaptive Thresholding

5. Postprocessing

As result the signal is devided into active and inactive segments. Based on this segmentation two indices are defined.

$$
\begin{gathered}
\text { ActivityRatio }=\frac{\sum \text { length of all active sections }}{\text { overall length }} \\
M L A S=\frac{\sum \text { length of all active sections }}{\text { Number of active sections }} \\
\text { III. RESULTS }
\end{gathered}
$$

\section{RESUltS}

\section{A. Test of NLEO with Coronary Sinus Data}

To test the two indices defined in (3) and (4) they are applied on a data set measured in the coronary sinus. The data was recorded during sinus rhythm, atrial flutter and atrial fibrillation over a time of 60 seconds from the same person. Five segments with a length of 6 seconds are analyzed and the mean ActivityRatio and MLAS were computed (table 1). 


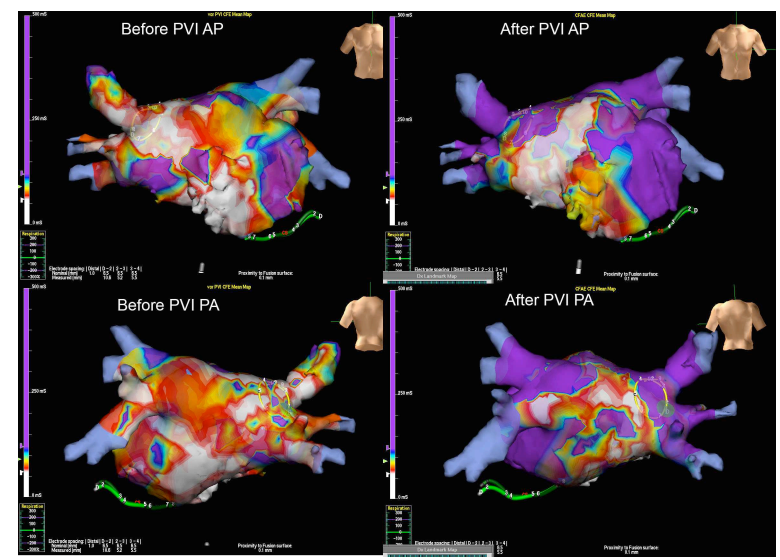

Fig. 1: CFAE Map before and after Pulmonary Vein Isolation (EnSite NavX ${ }^{\mathrm{TM}}$ ). White areas: high fractionation, purple areas: low fractionation.
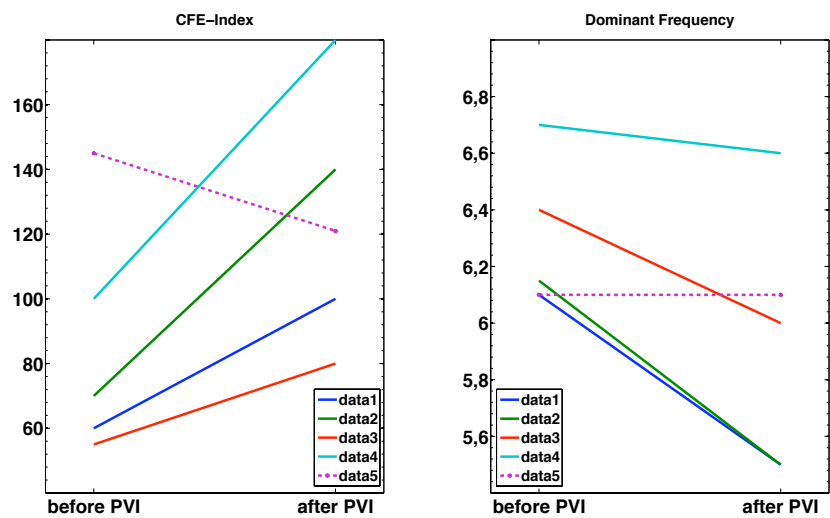

Fig. 2: Mean CFE-Index (EnSite NavX ${ }^{\mathrm{TM}}$ ) and dominant frequency before and after Pulmonary Vein Isolation. data1-data4 terminated during ablation, data5 did not terminate.

Table 1: Fractionation in the Coronary Sinus

\begin{tabular}{lcc}
\hline & ActivityRatio & MLAS \\
\hline Sinus Rhythm & 0.11 & 50.16 \\
Atrial Flutter & 0.27 & 59.38 \\
Atrial Fibrillation & 0.42 & 74.03 \\
\hline
\end{tabular}

Comparing the three states, it is evident that the ActivityRatio is increasing when the disarrangement of the excitation propagation from sinus rhythm to AF rises. That means the length of the active segments is increasing and also the number of fractionated signals. The data is getting more and more irregular.

The MLAS delivers similar results. It is also increasing when the electrogram gets disarranged.

\section{B. A Patient Study}

To investigate the clinical value of the ActivityRatio and MLAS, they are compared to clinically proved methods (CFE-Index, DF). The discribed methods were compared on data sets of 5 patients. Complex fractionated electrograms (CFAE) were recorded before and after Pulmonary Vein Isolation (PVI) at defined positions in the left atrium (roof, septum, anterior and posterior wall) using the Ensite NavX System. For all data sets the mean CFE-Index, the dominant frequency (DF), ActivityRatio and MLAS were calculated.

Out of the five analyzed data sets four were from patients who terminated during catheter ablation (data 1-4, 3x direct termination, $1 \mathrm{x}$ via an atrial tachycardia). The fifth data set was from a patient who underwent external cardioversion. In those patients who could be terminated by catheter ablation the mean CFE-Index increased from 71,25 $\pm 21,1 \mathrm{~ms}$ before to $125 \pm 44,3 \mathrm{~ms}$ after PVI (indicating decreasing fractionation!) and the mean DF decreased from $6,4 \pm 0,3 \mathrm{~Hz}$ before to $5,9 \pm 0,5 \mathrm{~Hz}$ after PVI. Analyzing the patient without termination of $\mathrm{AF}$ the mean CFE-Index decreased (145 ms before and $125 \mathrm{~ms}$ after PVI) and the DF did not change at all.

The same result is given by calculating the ActivityRatio and the MLAS. For the data sets with termination of AF the ActivityRatio decreased from 0.67 before PVI to 0.46 after PVI and the MLAS from 243.2 to 125.2 (indicating decreasing fractionation).

The values for the data set without termination are increasing from 0.49 to 0.67 (ActivityRatio) and 128.8 to 176.0 (MLAS).

\section{CONCLUSION}

The new approach for locating CFAEs works robust and can be used as bases of a following signal analysis. It does not have the disadvantages of the dominant frequency which is only reasonable for a subset of CFAEs [9]. Also it is not limited to a specific signal length like the CFE-Index. But the main disadvantage of the CFE-Index is that it is depending on a long list of settings which have to be defined by each cardiologist individually. So the result is not unique. Using adaptive thresholding the new algorithm is less userdependent. Filtering and baseline wander removal is essential to get good results. The two new indices (ActivityRatio and MLAS) reflect the disarrangement of the excitation propagation when the heart rhythm evolves from sinus rhythm to atrial fibrillation. Compared with CFE-Index and DF they are well-defined, user-independent and they give the same information on analyzing CFAEs before and after pulmonary vein isolation. 

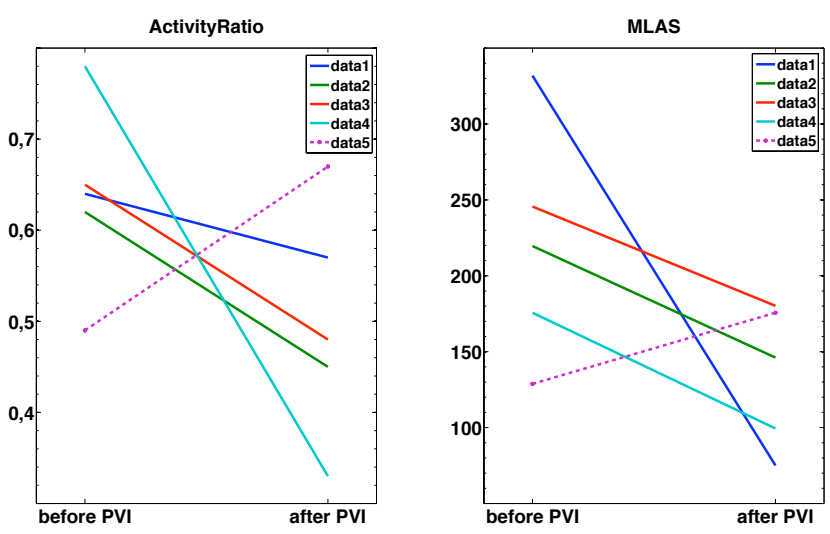

Fig. 3: Mean ActivityRatio and mean MLAS before and after Pulmonary Vein Isolation. data1-data4 terminated during ablation, data5 did not terminate.

Based on the segmented data further analysis like timefrequency analysis can be performed to get a better understanding of the electrophysiological processes.

\section{REFERENCES}

1. Wright M., Haissaguerre M., Knecht S., et al. (2008) State of the art: catheter ablation of atrial fibrillation. Journal of Cardiovascular Electrophysiology 19:583-592.

2. Schmitt C., Estner H., Hecher B., et al. (2007) Radiofrequency ablation of complex fractionated atrial electrograms (CFAE): preferential sites of acute termination and regularization in paroxysmal and persistent atrial fibrillation. Journal of Cardiovascular Electrophysiology 18:1039-1046.

3. Oral H., Chugh A., Good E., et al. (2007) Radiofrequency catheter ablation of chronic atrial fibrillation guided by complex electrograms. Circulation 115:2606-2612.

4. Takahashi Y., O'Neill M.D., Hocini M., et al. (2008) Characterization of electrograms associated with termination of chronic atrial fibrillation by catheter ablation. Journal of the American College of Cardiology 51:1003-1010.

5. Nguyen M.P., Schilling C., Dössel O. (2009) A new approach for automated location of active segments in intracardiac electrograms. submitted to the 11th International Congress of the IUPESM .

6. Kaiser J.F. (1990) On a simple algorithm to calculate the 'energy' of a signal. Acoustics, Speech, and Signal Processing, 1990. ICASSP-90., 1990 International Conference on :381-384.

7. Agarwal R., Gotman J. (1999) Adaptive segmentation of electroencephalographic data using a nonlinear energy operator. Circuits and Systems, 1999. ISCAS 4:199-202.

8. Khawaja A. (2006) Automatic ECG analysis using principal component analysis and wavelet transformation. Universitätsverlag, Karlsruhe.

9. Jason NG, Goldberger Jeffrey J. (2007) Understanding and interpreting dominant frequency analysis of AF electrograms. Journal of Cardiovascular Electrophysiology 18:680-685.

- Author: Christopher Schilling

- Institute: Institute of Biomedical Engineering, Universität Karlsruhe $(\mathrm{TH})$

- Street: Kaiserstrasse 12

- City: 76131 Karlsruhe

- Country: Germany

- Email: Christopher.Schilling@ibt.uni-karlsruhe.de 